\title{
Diritto Internazionale e Diritto dell'Integrazione in America Latina
}

\author{
Vicente Marotta Rangel \\ Diretor da Faculdade de Direito da Univer- \\ sidade de São Paulo e Catedrático de Direito \\ Internacional Público da mesma Faculdade

\section{Gustavo Zanini} \\ Professor Assistente Doutor da Faculdade \\ de Direito da Universidade de São Paulo
}

Furono i primi contatti degli europei con gli abitanti del Nuovo Mondo (in quelle regioni che poi costituirono l'America Latina) che provocarono il sorgere di un corpo sistematico di principi e norme tendendo a regolare le relazioni fra popoli diversi: il diritto internazionale.

La natura delle relazioni fra, da un lato, gli indigeni e dall'altro, spagnoli e portoghesi; il bisogno o meno di un ordinamento giuridico specifico che disciplinasse quelle relazioni; i problemi decorrenti dal contatto fra popoli di civiltà diverse - ecco le questioni suscitate dai filosofi, teologi e giuristi del XV e XVI secoli. Le loro risposte hanno dato i precetti basici alla nuova disciplina giuridica, che Francisco de Vitoria - tenendo conto della definizione di Gaio, pur alterandola - denominò «diritto delle genti» (1).

Nonostante fosse da molto presentita, ciò che spiega l'affermazione paradossale secondo cui l'America prima di essere scoperta era stata inventata, fu la realtà della coscenza delle nuove terre e dei nuovi popoli il fenomeno catalizzatore della gestazione e consolidazione di una nuova disciplina.

Sin dal princípio, il continente scoperto era destinato a stimolare il sorgere e lo sviluppo di norme e istituzioni; il che non è occorso in

Trabalho apresentado no Seminário Internacional de Estudos «Europa Comunitária e América Latina: Integração e Cooperação Econômica», realizado em Salerno (Itália), de 28 a 30 de abril de 1983.

(1) Si veda al riguardo, anche per estesi riferimenti bibliografici: Massimo Panebianco, «La recezione del «ius gentium «in Europa e in America Latina», in Rivista di Diritto Internazionale Privato e Processuale, n. 2, 1981, p. 434 ss. 
completa armonia con quelle. Tali discrepanze sebbene non frequenti hanno concorso a stimolare la revisione e il perfezionamento del diritto internazionale.

Al momento della conquista, l'America Latina e i suoi popoli hanno un rapporto col diritto internazionale che si puo definire naturale insito nella cultura del dominatoré.

Tre secoli più tardi circa, con l'emancipazione dei paesi del Nuovo Mondo, sono i giuristi ivi nati che imprimono un segno specifico al diritto internazionale in quel tempo vigente.

Fra quei due momenti però, non tutto si è verificato senza la presenza di quel segno, malgrado essafosse sorta in forma indiretta e isolata. Cosi, essa si è presentata nel Trattado di Madrid, del 13 gennaio 1750, firmato tra Don João V, re di Portogallo e Don Fernando VI, re di Spagna. D'altronde, l'articolo 21 di questo Trattato stipulava che, nel caso scopiasse una guerra fra Spagna e Portogallo, «i loro vassali stabilitisi in tutta l'America Meridionale, avrebbero dovuto mantenersi senza ostilità fra essi e i loro alleati»(2).

Un altro principio rilevante stabilito nel Trattato di Madrid è quello della applicazione dell'istituto di diritto privato dell' uti possidetis nell'ambito del diritto internazionale. Menrte il Preambolo del Trattato afferma che «ogni parte rimarrà con quello che attualmente possede», l'articolo III consagra chiaramente il principio dell «uti possidetis» ${ }^{(3)}$.

Entrambi gli aspetti rilevanti del Trattato di Madrid confermano la formazione graduale in America Latina di interessi e norme specifiche del Nuovo Mondo, le quali avevano ragione di essere, monostante fossero avversati nella Madre Patria.

Sin dal XVII secolo si accentuò nel continenté la firma di trattati internazionalli; il che aumentò il prestigio del diritto positivo cosi come le concezioni positiviste di questo diritto. Le concezioni giusnaturaliste, a loro volta, subirono modificazioni appartandosi dalle basi obbiettive presenti negli scritti di Tommaso d'Aquino, Vittoria o Suarez. Il diritto internazionale cerca di concentrarsi nel circolo dei paesi europei e a riflettere in qualche modo, gli interessi immediati. Ignorate gradualmente le concezioni giusnaturalisti che, nel corso del XVI secolo, furono la base e le origini del diritto delle genti e ligittimarono, su basi giuridiche, i rapporti reciproci tra gli europei e gli indigeni americani loro sudditi, il diritto internazionale tra i secoli XVII e XIX, propendeva a riflettere gli interessi oligarchici degli Stati Europei, le cui relazioni reciproche si proponevano di reggere.

(2) Jose Carlos de Macedo Soares: Fronteiras do Brasil no Regime Colonial. Rio de Janeiro, J. Olympio, 1939, p. 135. - Siveda peraltro: Rodrigo Octavio, «Alexandre de Gusmão e o Monroismo», RIHGB, 1941, vol. 175, p. 24-25.

(3) Jose Carlos de Macedo Soares, op. cit., p. 136. - Si veda anche: Pedro Calmon, Historia do Brasil, p. 119. 
Anche gli altri continenti erano soggetti ad un processo di occupazione e di colonizzazione da parte di questi Stati. Questi erano i principi, accolti dal diritto internazionale di quell'Epoca che si chiamava anche diritto pubblico della Europa o ius publicum Europaeum, denominazione che si trova nei libri de Johann Jacob Moser (1701-1785), Versuch des neuesten enropäischen Völkerrechts in Friedens und Kriegszeiten; e di Georg Friederich von Martens (1756-1821), Primae Lineae iuris gentium Europaearum practici.

Il sorgere delle nazioni Latino-americane verso una vita indipendenti ha indotto i suoi giurisconsulti a percepire il bisogno di approfondire gli studi di diritto internazionale.

Difatti, la situazione dei Nuovi Stati, che nón avevano ancora raggiunto un grado di sviluppo notevole data la loro penosa lotta per acquistare l'indipendenza - esigeva una definizione più precisa sui loro diritti nei confronti dei paesi dotati di sostanziali e solide ricorse proprie, poichè non potevano garantire soddisfacentemente quei diritti con il simplice potere delle armi ${ }^{(4)}$.

La prima opera divulgata in America Latina che, in modo metodico, contiene $\mathrm{i}$ principali aspetti del diritto internazionale, risale al 1832: «Princípios de derecho de gentes», di Andrés Bello (1781-1865).

Nato a Caracas, Andrés Bello visse anche a Londra (1810-1829), da dove si trasferi in Cile, nel qual paese, dopo più di trentanni di studi e di insegnamento, mori. Di formazione umanista, lettore di Omero e Sofocle, poeta giornalista, incaricato dal 1833, di elaborare il Progetto del Codice Civile Cileno (poi, Legge di 1855), filosofo, diplomatico, filologo ha scritto su diversi aspetti del diritto, soprattutto il romano, la filosofia del diritto, il diritto civile i il diritto delle genti.

Malgrado la formazione culturale del giurista latino-americano fosse strettamente ortodossa, nelle sue opere sono evidenti l'amore e la fede nella sua terra e nelle sui genti in un periodo particolarmente difficile com' è quello dell'emancipazione e di consolidamento dell'indiferenza dei paesi del Nuovo Mondo, il che equivale a un atto rivoluzionario. rilievo.

In determinati temi specifici questo caratteré è posto in singolare

Il principio dell'eguaglianza degli Stati fu uno di questi temi. «Siendo los hombres naturalmente iguales, lo son tambien los agregados de hombres que componen la sociedad universal. La republica más débil goza de los mismos derechos y está sujeta a las mismas obligaciones que el imperio más poderoso» ${ }^{(5)}$.

(4) Si veda a questo riguardo: Juan Carlos Puig, Derecho de la Comunidad Internacional, vol. I, Buenos Aires, 1975, pp 61-62.

(5) «Principios de Derecho de Gentes, Santiago de Chile, 1832, in Obras completas de Andrés Bello, X, p. 31 . 
Dopo aver citato Phillimore e Vattel (1714-1767), i quali seguono la stessa dottrina, Andrés Bello ricorda l'eccezione sollevata da Wheaton, nel capitolo Dei Diritti dell'Eguaglianza del suo libro Elementos, secondo cui «l'eguaglianza naturale degli Stati sovrani può modificarsi a causa di un contratto positivo o del costume, la qual cosa crea una posizione di superiorità da parte di uno Stato sugli altri per quanto riguarda la categoria, il titolo e altre distinzioni relative al cerimoniale.» Il capitolo di Weaton secondo Andrés Bello dovrebbe essere chiamato più propriamente: «Dei Diritti della diseguaglianza ${ }^{(6)}$.»

Circa le dottrine tradizionali di diritto internazionale, la posizione di Andrés Bello può essere qualificata di rinnovamento nella continuità. "Siamo incorporati», - scrisse - «in una grande associazione di popoli, della cui civiltà la nostra è un raggio. L'indipendenza che abbiamo acquistato ci ha messo in contatto immediato con le nazioni più sciluppate e colte, nazioni ricche di conoscenza, delle quali possiamo far parte con il solo fatto di desiderarlo. Tutti i popoli che figurarono prima di noi nella scena del mondo, hanno, lavorato per noi ${ }^{(7)} . \gg$

Cinque anni prima della pubblicazione dei Principios di Andrés Bello, cominciarono in Brasile $i$ corsi giuridici con due Scuole di Diritto: una al Nord, a Olinda, l'altra al Sud, a São Paulo. Titolare di questa Scuola di una cattedra comprendeva fra le altre discipline anche quella del diritto delle genti, José Maria de Avelar Brotero, pubblicò nel 1836, «Questioni su Prede Marittime.» Questa Monografia si ispira non soltanto a Grozio ma anche alla ricca bibliografia sul diritto delle genti in generale e sul diritto internazionale marittimo, come si può vedere nell'edizione del 1863. Dalla bibliografia constano i Trattati classici di Wolff e di Vattel tra gli altri.

Se dal lettore dell'Accademia di São Paulo sorse, cronologicamente, la prima monografia sul diritto delle genti, fu dal professore dell'Accademia di Olinda che si originò il primo riassunto sulla disciplina pubblicato a Pernambuco, nel 1851. Si tratta degli «Elementos de Direito das Gentes segundo as doutrinas dos autores mais modernos,» di Pedro Autran da Matta Albuquerque.

Sia nel riassunto che nella monografia del professore di São Paulo c'è una larga parte dedicata al diritto della guerra, al «ius belli», che il progresso della società ha ridimensionato, assegnandogli un posto di minore importanza nei programmi di diritto internazionale.

La maggior parte dei nostri primi giuristi si formò alla Scuola delle citate opere acquisendo per mezzo di esse gli strumenti essenziali per la difesa del diritto delle genti; mi riferisco concretamente a Joaquim Nabuco, a Rui Barbosa e al Barone di Rio Branco.

(6) «Principios», Ibidem, nota n. 1.

(7) «Principios», op. cit., p. 34. 
Pubblicato in Castigliano a Parigi, indue volume, vede la luce Le Droit Internacional Théorique et Pratique, la cui marca latina mira alla dottrina conosciuta solo attraverso il nome del suo autore, Carlos Calvo (1829-1906), dottrina presentata nella nota diplomatica che lui stesso formulò, in qualità di Ministro degli Affari Esteri della Repubblica Argentina, il 29 dicembre 1902 e presentata al Governo degli Stati Uniti d'America. Tale dottrina, come segnalò Rui Barbosa nel corso della Seconda Conferenza dell'Aia, un anno dopo il decesso del giurista argentino, agì poderosamente nello sviluppo dell'indipendenza dell'America ${ }^{(8)}$.

Soltanto alla fine del secolo scorso nasce la qualifica di «americano» aggiunta al cosi detto diritto internazionale europeo; ciò é sucesso grazie all'opera di P. Pradier-Fodéré, il «Traité de Droit Internacional Public Européen et Américain» i cui nove volumi furono pubblicati tra 1885-1905.

A proposito della denominazione del diritto internazionale diceva Lafayette Rodrigues Pereira, agli inizi di questo secolo, avrebbe dovuto essere «correttamente» chiamato «Diritto Pubblico Esterno Europeo-Americano ${ }^{\left({ }^{()}\right.}$.»

«La fase attuale dell'integrazione economica latino-americana non è niente altro che il culmine di un processo le cui radici coincidono, cronologicamente, con quelle della stessa identità nazionale delle Repubbliche Latino-americane» ${ }^{(10)}$. Al contrario di quello che è sucesso negli Stati Uniti d'America, la cui formazione si strutturò nella tendenza centrípeta a cui si sottomisero le antiche tredici colonie della Corona Britannica nell'America Latina predominò una tendenza opposta, centrifuga, giacchè i vicereami spagnoli si divisero in numerosi paesi indipendenti e stremamente gelosi della loro sovranità nazionale. Tali tendenza antagoniche diventano comprensive dato che c'era una predominante omogeneità culturale, linguistica, religiosa, giuridica tra le antiche colonie inglesi mentre l'eventuale omogeneità fra i nuovi paesi latino-americani era fortemente contrastata di uno svariato grado di incrocio di razze, culture indiane distinte, condizioni ecologiche diverse nei continenti e nelle isole, negli altipiani, nelle pianure, nelle economie agrarie e pastorali $o$ in quelle minerarie.

Un altro punto che merita di essere segnalato in questo ordine di idee è quello che si riferisce al periodo di dominazione napoleonica. In questa fase storica, infatti, si è presentata l'opportunità ai paesi latino-americani, di svincolarsi dai conquistatori portoghesi e spagnoli onde poter sussistere politicamente independenti. Va aggiunto, d'altra

(8) Si veda a questo proposito: Albert de la Pradelle - Maitres et Doctrines du Droit des Gens, 2s Ed. Paris, Les Editions Internationales, 1950, p. 217.

(9) Lafayette Rodrigues Pereira - Principios de Direito Internacional, I, Rio de Janeiro, 1902, p. 22.

(10) Derecho de la Integración Latino-Americana. Instituto Interamericano de Estudios Juridicos Internacionales. Buenos Aires. Depalma, 1969, p. 429, nota 1. 
parte, che l'idea dell'auto governo, seguita da una effettiva sovranità territoriale costituisce la base per affrontare qualsiasi tentativo di riconquista da parte di paesi europei. $\dot{\mathrm{E}}$ appunto in vista di questa situazione che non è difficile rendersi conto del naturale sorgere di un sentimento di nazionalismo assai accentuato. Sotto questo riguardo si aggiunga che, com'è del tutto comprensibile, anche l'auto governo diventava uno degli obbiettivi principali dei paesi latino-americani ${ }^{(11)}$.

Uomini di grande visione, stattisti con una prospettiva storica forse prematura per la loro epoca, sentirono i pericoli del «caudillismo» governo fatto a somiglianza delle anfizionie greche, che cercarono di ristabilire le grandi unità geopolitici-economiche ereditate dal periodo coloniale. Tali uomini furono San Martin, Alberdi, Miranda, Del Valle, Monteagudo, O'Higgins, Artigas e soprattutto Simón Bolivar, la cui visione forse profetica di una confederazione latino americana fu il principale fattore stimolante del movimento a favore dell'nità continentale, e che fece di lui il grande precursore dell'integrazione regionale. Questa visione spinse Bolivar a convocare il Congresso di Panamá che si reunì dal 22 giugno al 15 lugio 1826.

Il Congresso di Panamá ebbe lo scoppo di stabilire, tramite le vie commerciali, la confederazione perpetua delle nazioni americane. La concezione bolivariana dell'unità continentale aveva come principio basico quello della reciprocità degli interessi deu Nuovo Continente.

Nello stesso anno in cui terminò la riunione di Montevedeo, si realizzò a Washington, dal 2 ottobre 1889 al 18 aprile 1890, la Prima Conferenza Internazionale Americana, a cui parteciparono delle delegazioni di tutti i paesi americani, tranne la Repubblica Dominicana. La circolare del Segretario di Stato degli Stati Uniti, Bayard, del 13 luglio 1888, che invitava gli altri paesi americani al Congresso di Washington, menzionava, tra gli argomenti da discutere, c'erano «le misure destinate alla formazione di un'unione doganale americana, che incoraggiasse, mentre era possibile e vantaggioso, il commercio reciproco tra le nazioni dell'emisfero americano». La visione di Blaine, Segretario di Stato, attento alla realtà economica dell'industria nordamericana che aveva bisogno di mercanti di consumo cosi come di matrie prime tropicali, può far pensare alla creazione nell'Emisfero Occidentale, di un'unione doganale simile al Zollverein tedesco, chiave e elemento propulsore dell'egemonia prussiana e dell'unificazione della Germania (12).

Stabilitasi nel 1948, la CEPAL (Commissione del Consiglio Economico e Sociale per l'America Latina) esercitò una funzione decisiva nella creazione e nel progresso del mecanismo di integrazione latinoamericano. Un anno dopo la sua instituzione aveva già pronosticato la necessità di una coordinazione regionale delle politiche nazionali di sostituzione e di importazioni, difendendo cosi l'intercambio commer-

(11) Idem, p. 435.

(12) Idem, Ibidem, p. 435. 
ciale intralatinoamericano. Il suo Comitè di commercio preparò a questo riguardo diversi progetti che aspiravano a stabilire un mercato comune e uno stesso sistema di pagamento. Nel terzo Periodo di Sessione (1950), la CEPAL approvò una decisione con cui raccomandava ai Gruppi latino-americani di prendere in considerazione «le possibilità di espansione di domanda mediante lo scambio reciproco allo scopo di sfruttare una migliore integrazione delle economie e un più elevato sviluppo della produttività e della sua efficienza (Conf. Doc. E/CN, 12/194). Inoltre, nel suo quarto Periodo di Sessione (1951), 1. CEPAL stabilì i fondamenti del Programma per l'integrazione Centro-americana e servi come Segreteria Tecnica per il Comité di Cooperazione Economica dell'Istmo Centro-americano.

Innumerevoli fattori diedero origine agli argomenti economici in favore dell'integrazione:

1 - La reazione dell'Americana Latina tenendo conto delle sempre maggiori differenze tra i livelli di sviluppo ed il benessere del mondo e lo stato di crisi periodiche in cui cadeva l'economia della regione;

2 - Lo scontento condiviso in grado crescente, da altre regioni sottosviluppate;

3 - La certezza che, nel mondo attuale, costituito da gruppi politici ed economici la conquista del potere dei paesi sottosviluppati dipendesse dalla possibilità di unificazione dei suoi criteri e dall'orientazione dei centri industriali (13).

Il processo di integrazione economica dell'America Centrale obbedi a successive tappe, la cui più antica, la preliminare, si estese dal 1951 al 1957 nel corso delle convenzioni bilaterali celebrate tra paesi che influirono nel contenuto dei primi trattati collettivi di integrazione: il Trattato Multilaterale di Libero Commercio e Integrazione e il Trattato Generale di Integrazione Economica Centro-americano, relativi rispettivamente a Tegucigalpa (1958) e a Managua (1960).

Per quanto riguarda il Trattato Generale di Integrazione Economico Centro-Americano, del 13 dicembre 1960, si tratta dello strumento più importante sul funzionamento del Mercato Comune di questa regione istmica, nonostante non possa essere applicato nella sua pienezza, in questi giorni, data la crisi provocata dai fattori di ordine politico, di qualche paese firmatorio del Trattato, cosi como la crisi economica mondiale che essi affrontano.

Cosi come in America Centrale, in cui il movimento rimase circoscritto strettamente alle frontieri geografiche, l'idea iniziale fu quella di integrare il considetto Cono Sud dell'America Latina, come tappa preliminare di una associazione più ampia.

(13) Miguel S. Wionezek: «Introdução. Requisitos para uma Integração», in Latin American Economic Integration. Friederich A. Praeger (tradução Brasileira), Rio de Janeiro, 1966, O Cruzeiro, 1966). 
Tale proposito di limitazione regionale fu, però, respinta dalla partecipazione del Messico in primo luogo, e in seguito da quella della Colombia e dell'Ecuador ${ }^{(14)}$. D'altra parte, l' elezione di formula di una zona di libero commercio ebbe il suo fondamento in motivi politici e pratici. I tecnici pensavano che non fosse possibile convincere $i$ governi e i parlamenti della necessità di consacrace giuridicamente, sin dal primo momento, l'istallazione di una unione doganale.

La Conferenza di Montevideo approvò, in febbraio del 1960, le sole basi di integrazione che avrebbero potuto essere accettate in quell'epoca, dai rispettivi governi (15).

Sottoscritto a Montevideo, il 18 febbraio del 1960, il Trattato costitutivo dell'Associazione Latino-Americana di Libero Commercio (ALALC) prevedeva che la zona di libero commercio da lui istituita avrebbe dovuto perfezionarsi entro un periodo non superiore a dodici anni calcolato a partire della sua messa in vigore ${ }^{(16)}$.

Tale periodo fu protratto fino al 31 dicembre 1980, nei termini del Protocollo di Caracas, firmato il 12 dicembre 1969, nella capitale del Venezuela. Le Parti (Argentina, Bolivia, Brasile, Cile, Colombia, Ecuador, Messico, Paraguai, Perù, Uruguai e Venezuela) si erano impegnati a eliminare, gradualmente, per quanto riguardava l'essenziale del loro commercio reciproco, i gravami e le restrizioni che incidevano sull'importazione dei prodotti originari del territorio dí qualsiasi Contratante. Tale eliminazione si sarebbe raggiunta tramite le trattative periodiche di cui avrebbero dovuto risultare:

a) Cataloghi nazionali, con le riduzioni annuali di gravami e altre restrizioni che ogni Parte decideva di accordare alle altri Parti;

b) Un Catalogo comune, con la descrizione dei prodotti i cui gravami e restrizioni, le Parti si impegnavano, per decisione colletiva, ad eliminare integralmente dal commercio intrazonale. L'eliminazione di barriere commerciali intraregionali era considerata, secondo il Preambolo, un mezzo di espansione dei mercati nazionali e condizioni per accelerare il processo e lo sviluppo economico dei paesi dell'America Latina.

$\mathrm{Ci}$ sono delle ragioni rilevanti che indussero l'America Latina a non riuscire como il Mercato Comune Europeo:

1 - il Trattato di Roma fu firmato in condizioni molto favorevoli, e cioè, durante un periodo di sviluppo assai rapido dell'Europa Occidentale. Il Trattato di Montevideo invece fu fatto in un periodo di recessione;

(14) Gustavo Margariños: «Instrumentos da Integração e Realizações da ALALC). A Integração Econômica da América Latina, p. 145.

(15) Idem, p. 146.

(16) «En realidad el Tratado no establece una Zona de Libre Comercio, sino un Convenio Programado para llegar a una Zona en 12 años, conforme al articulo XXrV dell GATT. (in Javier Silva Barros: Régimen Legal de los Acuerdos de Complementación en la ALALC). - Derecho de la Integración, n. 5, 1969, p. 80). 
2 - le differenze di livelli di sviluppo economico tra i paesi dell' ALALC furono bem maggiori di quelli esistenti tra qualunque altro paesi dell'Emisfero nord;

3 - per l'Europa Occidentale, la riduzione delle barriere interne, che resultarono dalla Grande Depressione e dalla Seconda Guerra Mondiale, costituivano un elemento artificiale nell'economia della regione, sicchè il programma di liberazione del commercio rappresentava un ritorno alla normalità. In America Latina, al contrario, si trattava di creare qualcosa che non era esistito anteriormente;

4 - il Trattato di Roma applica il rinnovo di tutte le restrizioni di commercio, e cioè, non solo di prodotti tradizionali ma anche di tutti gli articoli prodotti in qualsiasi parte dell'area del Mercato Comune. Il Trattato di Montevideo, al contrario, è redatto in modo da non rendere necessario per $\mathrm{i}$ paesi membri solo lo sviluppo del «commercio già esistente.»

5 - I paesi dell'Europa Occidentale nel proporre un programma di liberazione del Trattato di Roma furono molto più elastici dei paesi latino-americani, per ciò che riguarda i principi enunziati a Montevideo.

6 - Si suppone frequentemente che l'enfasi data dal MCE all'intensificarsi della concorrenza tra le industrie, con obbiettivo simile, è valida anche per l'America Latina. Nell'Europa Occidentale esiste già una industria compatta e il principale problema è quello di renderla più produttiva e efficiente. Lo sviluppo dell'America Latina si è verificato entro i limiti dei mercanti nazionali esistenti. Ciascuna delle industrie nazionali è positivamente orientata verso il potenziale del suo mercato e generalmente teme le conseguenze della concorrenza di rivali più efficiente (reali o in potenziale) dei paesi limitrofi, anzichè l'attesa paziente di mercati maggiori volta alla crezzione di un mercato comune regionale. Molto più importante è il problema che consiste nell'ampliare l'industria latino-americana oltre qualsiasi aspettativa, principalmente attraverso lo stabilirsi di industrie interamente nuove in settori dove ancora non ne esiste nessun'altra (17).

L'effetto favorevole che il Trattato di Roma ebbe sopra la crescita economica dell'Europa, Trattato firmato nel 1957, stimolò la conclusione del Trattato dell'ALALC, del 1960, il Trattato del Mercato Comune Centro-Americano del 1962 (o Trattato di Managua); nel 1969 l' Acordo di Cartagena; il Trattato dell'Associazione LatinoAmericana di Integrazione - (ALADI) -, del 12 agosto 1980. I

(17) Sidney S. Dell. «Os Primeiros Passos de Experiencia da ALALC», in A Integração Economica da América Latina. Editado por Miguel S. Wionezek. Rio de Janeiro (trad.) 1966, p. 129. 
paesi Latino-americani adottarono l'ideologia dell'integrazione delle Comunità Europee per i loro stessi sforzi di integrazione economica.

Allo stesso tempo combatterono energicamente determinati aspetti della nuova entità, in modo particolare la Política Agricola Comune (PAC) ${ }^{(18)}$.

\section{CONCLUSIONI}

Per le osservazioni storiche predette di carattere piuttosto analitiche, noi non abbiamo avuto sicuramente l'intenzione di esaminare il meccanismo convenzionale dei paesi Latino-americani, in tutta la complessità della sua struttura giuridica e economica e in tutte le sue strategíe nazionali di sviluppo.

Il nostro scopo non era penetrare nelle questioni specifiche su cui il Trattato dell'Associazione Latino-Americana di Integrazione (ALADI - 1980) cosi complesso e cosi nuovo regerà le relazioni economiche tra paesi del nostro continente.

D'altra parte noi abbiamo ritenuto necessario esaminare almeno nelle sue linee generali, la struttura del sistema giuridico economico prima di dedicarci a considerazioni di ordine più generale e astratto.

Considerare i paesi Latino-americani, dal punto di vista dell'integrazione economica vuol dire, anzitutto, ricercare quello che è il suo sistema politico e economico, come esso è nato e quali sono i suoi obbiettivi, tenendo conto della sua propria natura.

L'influenza del diritto internazionale europeo sul diritto internazionale latino-americano è stata profonda, tanto chè dopo un periodo di affermazioni proprie di autonomia, ancora oggi persistenti in quest'ultimo diritto - ambedue si identificano nelle loro norme e valori essenziali.

Per quanto riguarda il diritto di integrazione, tale influsso del diritto europeo fu egualmente sensibile alle origini del diritto di integrazione latino-americano ancorchè senza lo stesso carattere assoluto e esclusivista dell'impatto del diritto internazionale. Man mano che il tempo corre il modelo del diritto europeo di integrazione subisce delle modifiche a causa delle esigenze sociali e politiche specifiche dell'Americana Latina.

(18) Si veda in questo senso: Massimo Panebianco: «Dalla Societa delle Naziont all» O.N.U. (le grandi organizzazioni internazionali), Editrice Ferraro, 1977, p. 92 s. 